\title{
Lemierre's Syndrome: The Result of an Incorrect Penicillin Allergy
}

\author{
Christopher $\mathrm{D}^{1 *}$, Alyson $\mathrm{P}^{2}$ and Katherine $\mathrm{H}^{1}$ \\ ${ }^{1}$ University of Iowa Hospitals and Clinics, USA \\ ${ }^{2}$ University of Iowa Carver College of Medicine, USA
}

\begin{abstract}
We present a case of a previously healthy 21 year old male who presents with Lemierre's syndrome after getting inadequate treatment of a throat infection because of a penicillin allergy label. Lemierre's syndrome is a rare complication following an acute oropharyngeal infection due to the common use of penicillin in modern medicine. Unfortunately, many patients are labeled with a penicillin allergy at a young age and are therefore more susceptible to complications of not receiving the most effective treatment for, what should be, a routine illness.
\end{abstract}

\section{Case presentation}

A 21 y.o. male with no significant past medical history presents to the Emergency Room with shortness of breath and fever. Five days prior to presentation, he noted sore throat, fevers, and heartburn. Since that time, he has also noted increasing shortness of breath. He presented to an acute care clinic three days prior to presentation and had a positive rapid strep screen. He was given a script for azithromycin, due to a history of penicillin allergy, and magic mouthwash. He took Ibuprofen alternating with acetaminophen for fever, but continued having worsening dyspnea and a cough productive of clear sputum. He had significant pain over the right chest, $10 / 10$ in severity, sharp in quality, exacerbated by deep breathing.

In the ER his vital signs were significant for fever to $40.1 \mathrm{C}$, tachycardia, tachypnea, and hypoxia requiring $3 \mathrm{~L}$ oxygen. Physical exam revealed significant diaphoresis and decreased breath sounds at the right base. He had dullness to percussion over the right lower lung field, but no egophany was noted. A chest X-Ray showed possible right pleural effusion with no consolidation (Figure 1). CTA was obtained and showed no pulmonary emboli but found right air space concerning for pneumonia and an abscess superior to his right lower lobe bronchus. Blood cultures were obtained, he was given fluids and started on Ceftriaxone and Azithromycin (Figure 2).

On admission, Clindamycin was started for the concern of a pulmonary abscess until gram stains on the blood cultures grew Gram Negative and Gram Positive Rods. The patient was then converted to Ceftazidime, to cover possible pseudomonas, and Vancomycin, for possible MRSA. Over the next day, he continued to have significant right sided chest pain and persistent leukocytosis, fevers, and oxygen requirement. A repeat CXR was obtained on his third day of admission which showed a large right sided pleural effusion (Figure 3). Blood cultures at this time came back positive for Fusobacterium necrophorum which raised immediate concern for Lemierre's syndrome. He was restarted on IV Clindamycin and a CT larynx and chest was obtained, showing a possible non-loculated empyema, worsening infectious pneumonia, a clot in the left internal jugular vein, but no retropharyngeal abscess (Figures 4 and 5). The Cardiothoracic surgery team was consulted and the patient went for right sided Video Assisted Total Decortication. They were able to peel off an exudate made up of fibrinous material with focal granulation tissue surrounding the lung and then drain the fluid. He had 3 chest tubes to drain fluid over the next few days, and was discharged on post-op day 5. On follow-up 2 weeks later, he was still on clindamycin and was feeling better, with no fevers and much improved breathing. During admission, the allergy/immunology team found he did not have a true penicillin allergy.

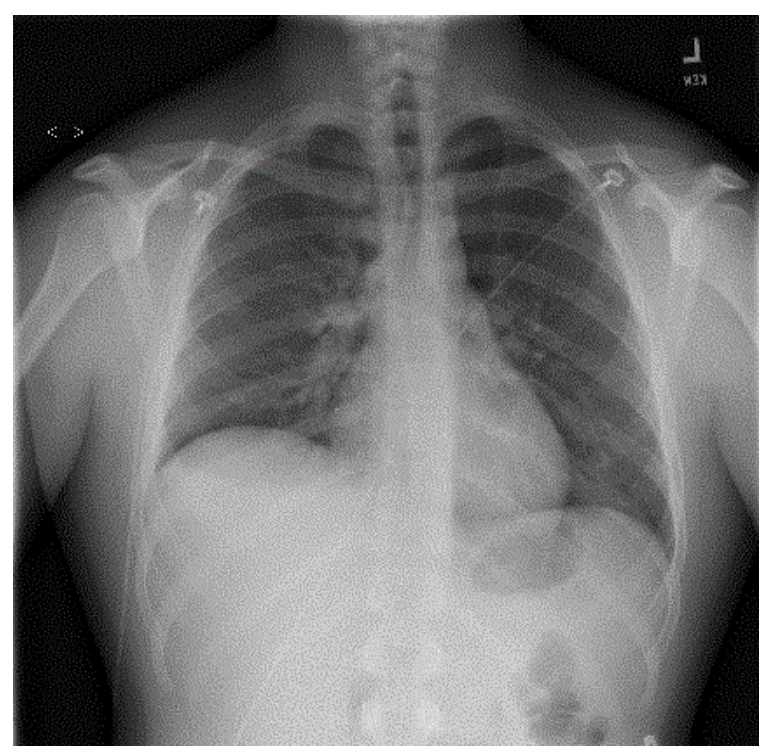

Figure 1: Chest X-Ray

Correspondence to: Christopher DeZorzi, Internal Medicine PGY-1, 1759 Louis Place, Iowa City, IA 52245, USA; E-mail: christopher-dezorzi@uiowa.edu

Received: May 02, 2017; Accepted: May 22, 2017; Published: May 25, 2017 


\section{Discussion}

Lemierre's Syndrome refers to the progression of a bacterial throat infection to thrombophlebitis of the internal jugular vein. This thrombophlebitis can have serious implications when it leads to bacteremia and septic emboli. Most commonly, and as in our case, the lungs are a site of metastatic infection. This can include pulmonary infiltrates, pleural effusion, empyema and lung abscesses [1-5]. Other metastatic infections include septic arthritis, osteomyelitis, pericarditis, hepatic abscesses, and meningitis. Other organs including the spleen, skin, kidneys, brain and soft tissues may also be involved [1,6-9].

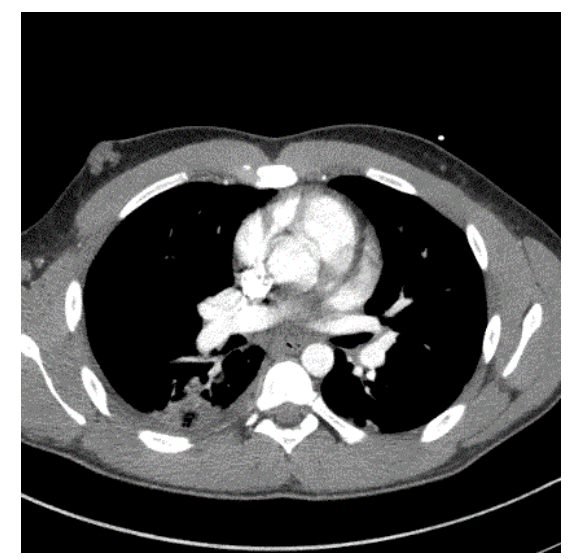

Figure 2. CTA

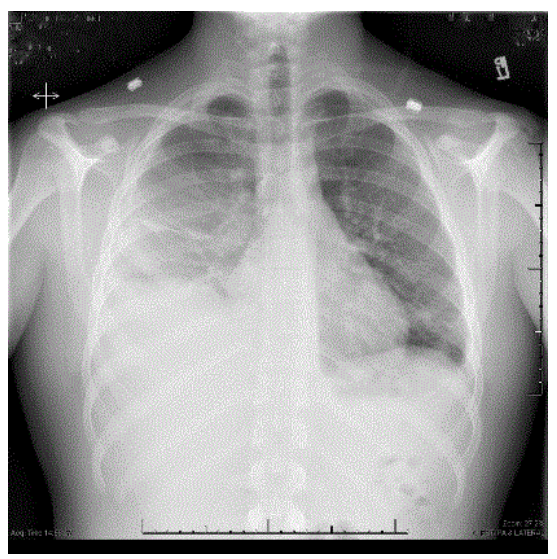

Figure 3. CXR

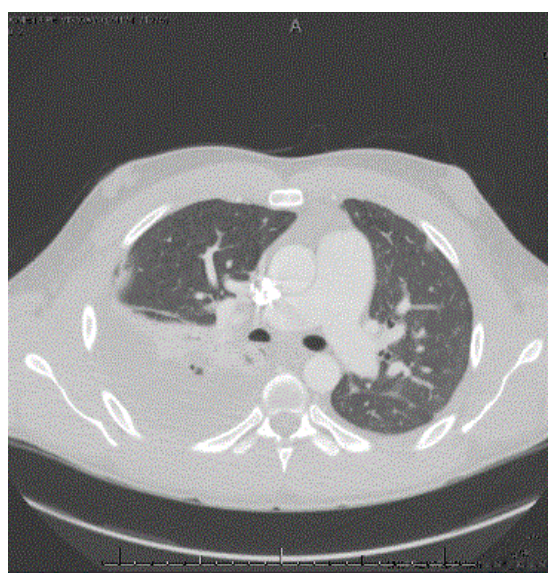

Figure 4. CT larynx

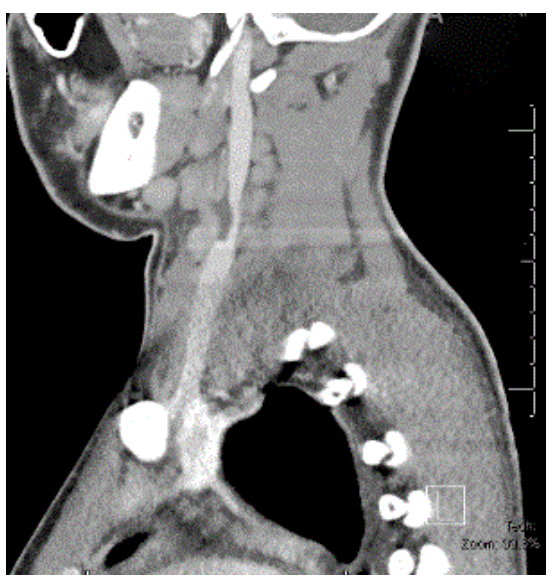

Figure 5: CT Neck

Lemierre's Syndrome is most commonly caused by Fusobacterium, which are normal flora of the oral cavity and gastrointestinal tract [10]. They are slow growing, anaerobic, gram-negative bacilli which are susceptible to Penicillin.

This constellation of symptoms occurs because a bacterial infection progresses to the formation of an abscess in the palatine tonsils or peri-tonsillar tissue. Inside the abscess, anaerobic bacteria can flourish. When this abscess ruptures, the bacteria can infect nearby structures including the internal jugular vein [10-12]. In most cases, this process is interrupted early in the course with the prescription of penicillin for strep throat. With increased use of penicillin for bacterial throat infections since the 1960s, the number of reports of Lemierre's syndrome has dropped [13]. Lemierre's syndrome remains a rare condition, with one retrospective study from Denmark estimating an incidence of around 1 case per million [14]. In our case, the patient had a charted "unknown allergy to penicillin," so he received Azithroymycin for a perceived strep throat infection instead of Penicillin. Fusobacterium species is much more likely to be resistant to Azithromycin than Penicillin. This "fake allergy" reporting has become an epidemic in our current healthcare system with serious consequences. In a recent study, $96 \%$ of patients at one acute care facility who self-reported penicillin allergy had a negative skin test [15]. Similar to those in this study, our patient was tested during this admission by the allergy/immunology team and found not to have a true allergy to Penicillin making his entire hospitalization preventable.

\section{Conclusion}

Lemierre's syndrome is a rare complication following an acute oropharyngeal infection due to the common use of Penicillin to treat strep throat in modern medicine. Unfortunately, many patients get labeled with a Penicillin allergy at a young age and are therefore more susceptible to complications of not receiving the most appropriate treatment for an illness such as strep throat. Primary Care providers should be more proactive in having an accurate allergy history and Hospitalists should be more suspicious of this syndrome in patients with a listed penicillin allergy.

\section{References}

1. Razonable RR, Rahman AE, Wilson WR (2003) Lemierre syndrome variant necrobacillosis associated with inferior vena cava thrombosis and pulmonary abscesses after trauma-induced leg abscess. Mayo Clin Proc 78: 1153-1156. [Crossref]

2. Cook RJ, Ashton RW, Aughenbaugh GL, Ryu JH (2005) Septic pulmonary embolism: presenting features and clinical course of 14 patients. Chest 128: 162-166. [Crossref] 
3. Sasaki Y, Iwata H, Kinoshita M, Sumiya M (2000) Lemierre syndrome with extensive cervical venous thrombosis and multiple pulmonary embolisms. Nihon Naika Gakkai Zasshi 89: 2174-2176. [Crossref]

4. Gormus N, Durgut K, Ozergin U, Odev K, Solak H (2004) Lemierre's syndrome associated with septic pulmonary embolism: a case report. Ann Vasc Surg 18: 243-245. [Crossref]

5. Shaham D, Sklair-Levy M, Weinberger G, Gomori JM (2000) Lemierre's syndrome presenting as multiple lung abscesses. Clin Imaging 24: 197-199. [Crossref]

6. Bentham JR, Pollard AJ, Milford CA, Anslow P, Pike MG (2004) Cerebral infarct and meningitis secondary to Lemierre's syndrome. Pediatr Neurol 30: 281-283. [Crossref]

7. Repanos C, Chadha NK, Griffiths MV (2006) Sigmoid sinus thrombosis secondary to Lemierre's syndrome. Ear Nose Throat J 85: 98-101. [Crossref]

8. Singaporewalla RM, Clarke MJ, Krishnan PU, Tan DE (2006) Is this a variant of Lemierre's syndrome? Singapore Med J 47: 1092-1095. [Crossref]

9. Alherabi A (2009) A case of Lemierre syndrome. Annals of Saudi Medicine 29: 58-60. [Crossref]
10. Brazier JS (2006) Human infections with Fusobacterium necrophorum. Anaerobe. 12: 165-172. [Crossref]

11. Huggan PJ, Murdoch DR (2008) Fusobacterial infections: clinical spectrum and incidence of invasive disease. Journal of Infection 57: 283-289. [Crossref]

12. Kushawaha A, Popalzai M, El-Charabaty E, Mobarakai N (2009) Lemierre's syndrome, reemergence of a forgotten disease: a case report. Cases Journal 2.

13. Leugers CM, Clover R (1995) Lemierre syndrome: postanginal sepsis. Journal of the American Board of Family Practice 8: 384-391.

14. Hagelskjaer LH, Prag J, Malczynski J, Kristensen JH (1998) Incidence and clinical epidemiology of necrobacillosis, including Lemierre's syndrome, in Denmark 19901995. European Journal of Clinical Microbiology and Infectious Diseases 17: 561-565. [Crossref]

15. Heil EL, Bork JT, Schmalzle SA, Kleinberg M, Kewalramani A, et al. (2016) Implementation of an Infectious Disease Fellow-Managed Penicillin Allergy Skin Testing Service. Open Forum Infect Dis 3: 155. [Crossref]

Copyright: $(\mathbb{C} 2017$ Christopher D. This is an open-access article distributed under the terms of the Creative Commons Attribution License, which permits unrestricted use, distribution, and reproduction in any medium, provided the original author and source are credited. 